\title{
Undecidability of the unification and admissibility problems for modal and description logics
}

\author{
Frank Wolter \\ University of Liverpool \\ and \\ Michael Zakharyaschev \\ Birkbeck College London
}

\begin{abstract}
We show that the unification problem 'is there a substitution instance of a given formula that is provable in a given logic?' is undecidable for basic modal logics $\mathrm{K}$ and $\mathrm{K} 4$ extended with the universal modality. It follows that the admissibility problem for inference rules is undecidable for these logics as well. These are the first examples of standard decidable modal logics for which the unification and admissibility problems are undecidable. We also prove undecidability of the unification and admissibility problems for $\mathrm{K}$ and $\mathrm{K} 4$ with at least two modal operators and nominals (instead of the universal modality), thereby showing that these problems are undecidable for basic hybrid logics. Recently, unification has been introduced as an important reasoning service for description logics. The undecidability proof for $\mathrm{K}$ with nominals can be used to show the undecidability of unification for Boolean description logics with nominals (such as $\mathcal{A L C O}$ and $\mathcal{S H I} \mathcal{I O}$ ). The undecidability proof for $\mathrm{K}$ with the universal modality can be used to show that the unification problem relative to role boxes is undecidable for Boolean description logics with transitive roles, inverse roles and role hierarchies (such as $\mathcal{S H I}$ and $\mathcal{S H I Q}$ ).
\end{abstract}

Categories and Subject Descriptors: F.4.1 [Mathematical logic and formal languages]: modal logic.

General Terms: theory.

Additional Key Words and Phrases: unification, admissible rule, description logic, hybrid logic, decidability.

\section{INTRODUCTION}

The unification (or substitution) problem for a propositional logic $L$ can be formulated as follows: given a formula $\varphi$ in the language of $L$, decide whether it is unifiable in $L$ in the sense that there exists a uniform substitution $s$ for the variables of $\varphi$ such that $s(\varphi)$ is provable in $L$. For normal modal logics, this problem is equivalent to the standard unification problem modulo equational theories [Baader and Siekmann 1994]: in this case the equational theory consists of any complete

Author's address: F. Wolter, Department of Computer Science, University of Liverpool, Liverpool L69 7ZF, U.K., frank@csc.liv.ac.uk. M. Zakharyaschev, School of Computer Science and Information Systems, Birkbeck College, London WC1E 7HX, U.K., michael@dcs.bbk.ac.uk.

Permission to make digital/hard copy of all or part of this material without fee for personal or classroom use provided that the copies are not made or distributed for profit or commercial advantage, the ACM copyright/server notice, the title of the publication, and its date appear, and notice is given that copying is by permission of the ACM, Inc. To copy otherwise, to republish, to post on servers, or to redistribute to lists requires prior specific permission and/or a fee.

(C) 2021 ACM 1529-3785/21/0600-0001 $\$ 5.00$ 
set of equations axiomatising the variety of Boolean algebras with operators and additional equations corresponding the axioms of $L$.

A close algorithmic problem for $L$ is the admissibility problem for inference rules: given an inference rule $\varphi_{1}, \ldots, \varphi_{n} / \varphi$, decide whether it is admissible in $L$, that is, for every substitution $s$, we have $L \vdash s(\varphi)$ whenever $L \vdash s\left(\varphi_{1}\right), \ldots, L \vdash s\left(\varphi_{n}\right)$. It should be clear that if the admissibility problem for $L$ is decidable, then the unification problem for $L$ is decidable as well. Indeed, the rule $\varphi / \perp$ is not admissible in $L$ iff there is a substitution $s$ for which $L \vdash s(\varphi)$.

It follows from the results of V. Rybakov (see [Rybakov 1997] and references therein; see also [Ghilardi 2000; 2004; Ghilardi and Sacchetti 2004; Iemhoff 2001; 2003]) that the unification and admissibility problems are decidable for propositional intuitionistic logic and such standard modal logics as K4, GL, S4, S4.3. However, nearly nothing has been known about the decidability status of the unification and admissibility problems for other important modal logics such as the ('non-transitive') basic logic K, various multi-modal, hybrid and description logics. In fact, only one - rather artificial - example of a decidable unimodal logic for which the admissibility problem is undecidable has been found [Chagrov 1992] (see also [Chagrov and Zakharyaschev 1997]).

The first main result of this paper shows that for the standard modal logics $\mathrm{K}$ and K4 (and, in fact, all logics between them) extended with the universal modality the unification problem and, therefore, the admissibility problem are undecidable.

The universal modality, first investigated in [Goranko and Passy 1992], is regarded nowadays as a standard constructor in modal logic; see, e.g., [Blackburn et al. 2006]. Basically, the universal box is an S5-box whose accessibility relation contains the accessibility relations for all the other modal operators of the logic. The undecidability result formulated above also applies to those logics where the universal modality is definable, notably to propositional dynamic logic with the converse; see, e.g., [Harel et al. 2000]. The unification and admissibility problems for $\mathrm{K}$ itself still remain open. Observe that $\mathrm{K} 4$ is an example of a logic for which the unification and admissibility problems are decidable, but the addition of the (usually 'harmless') universal modality makes them undecidable (although K4 with the universal modality itself is still decidable, in fact, PSPACE-complete). Note also that for 'reflexive' modal logics with the universal modality such as S4 the unification problem is trivially decidable.

The second result of this paper shows that the unification and admissibility problems are undecidable for multimodal $\mathrm{K}$ and $\mathrm{K} 4$ (with at least two modal operators) extended with nominals.

Nominals, that is, additional variables that denote singleton sets, are one of the basic ingredients of hybrid logics; see, e.g., [Areces and ten Cate 2006] and references therein. As follows from our second result, for most hybrid logics the unification and admissibility problems are undecidable.

A particularly interesting consequence of this result is in description logic. Motivated by applications in the design and maintenance of knowledge bases, Baader and Narendran [2001] and Baader and Kuesters [2001] identify the unification problem for concept descriptions as an important reasoning service. In its simplest formulation, this problem is equivalent to the unification problem for modal logics.

ACM Transactions on Computational Logic, Vol. V, No. N, June 2021. 
Baader and Narendran [2001] and Baader and Kuesters [2001] develop decision procedures for certain sub-Boolean description logics, leaving the study of unification for Boolean description logics as an open research problem. It follows from our results that unification is undecidable for Boolean description logics with nominals such as $\mathcal{A L C O}, \mathcal{A L C Q O}, \mathcal{A L C Q \mathcal { I O }}$, and $\mathcal{S H I Q O}$. Moreover, if a Boolean description logic has transitive roles, inverse roles and role hierarchies, then a role box can be used to define a universal role. In this case our results can be used to show the undecidability of unification relative to role boxes. This applies, for example, to the logics $\mathcal{S H \mathcal { I }}$ and $\mathcal{S H \mathcal { I }}$. These undecidability results cover almost all Boolean description logics used in applications, in particular the description logic underlying OWL-DL. However, the unification problem for some basic Boolean description logics such as $\mathcal{A L C}$ and $\mathcal{A L C} \mathcal{L I}$ remains open.

The plan of this paper is as follows. We start by introducing the syntax and semantics of normal modal logics with the universal modality, in particular $\mathrm{K} 4_{u}$ and $\mathrm{K}_{u}$. Then we prove, using an encoding of Minsky machines, the undecidability of the unification and admissibility problems for all logics between $\mathrm{K}_{u}$ and $\mathrm{K}_{u}$. We also briefly discuss the formulation of this result in terms of equational theories. Then we introduce modal logics with nominals and show how to modify the proof in order to establish the undecidability of unification and admissibility for $\mathrm{K}$ and $\mathrm{K} 4$ with at least two modal operators and nominals. We close with a brief discussion of consequences for description logics with nominals.

\section{UNIFICATION IN MODAL LOGICS WITH THE UNIVERSAL MODALITY}

Let $\mathcal{L}$ be the propositional language with an infinite set $p_{0}, p_{1}, \ldots$ of propositional variables, the Boolean connectives $\wedge$ and $\neg$ (and their derivatives such as $\vee, \rightarrow$, and $\perp$ ), and two unary modal operators $\square$ and $\forall$ (with their duals $\diamond$ and $\exists$ ). A normal modal logic $L$ with the universal modality $\forall$ is any set of $\mathcal{L}$-formulas that contains all propositional tautologies, the axioms

$$
\begin{aligned}
& \square(p \rightarrow q) \rightarrow(\square p \rightarrow \square q), \quad \forall(p \rightarrow q) \rightarrow(\forall p \rightarrow \forall q), \\
& \forall p \rightarrow p, \quad \forall p \rightarrow \forall \forall p, \quad p \rightarrow \forall \exists p, \quad \forall p \rightarrow \square p,
\end{aligned}
$$

and is closed under modus ponens, the necessitation rules $\varphi / \square \varphi$ and $\varphi / \forall \varphi$, and uniform substitution. $\mathrm{K}_{u}$ is the smallest normal modal logic with the universal modality. $\mathrm{K} 4_{u}$ is the smallest normal modal logic with the universal modality that contains the extra axiom $\square p \rightarrow \square \square p$.

$\mathrm{K}_{u}$ and $\mathrm{K} 4_{u}$ as well as many other normal modal logics with the universal modality are determined by relational structures. A frame for $\mathcal{L}$ is a directed graph $\mathfrak{F}=(W, R)$, that is, $R \subseteq W \times W$. A model for $\mathcal{L}$ is a pair $\mathfrak{M}=(\mathfrak{F}, \mathfrak{V})$ where $\mathfrak{F}$ is a frame and $\mathfrak{V}$ a valuation mapping the set of propositional variables to $2^{W}$. The truth-relation $(\mathfrak{M}, x) \models \varphi$ between points $x \in W$ of $\mathfrak{M}$ and $\mathcal{L}$-formulas $\varphi$ is defined inductively as follows:

$$
\begin{aligned}
(\mathfrak{M}, x) & \models p_{i} \text { iff } x \in \mathfrak{V}\left(p_{i}\right) \\
(\mathfrak{M}, x) & \models \neg \psi \text { iff }(\mathfrak{M}, x) \not \psi \psi \\
(\mathfrak{M}, x) & \models \psi \wedge \chi \text { iff }(\mathfrak{M}, x) \models \psi \text { and }(\mathfrak{M}, x) \models \chi \\
(\mathfrak{M}, x) & \models \square \psi \text { iff }(\mathfrak{M}, y) \models \psi \text { for all } y \in W \text { with } x R y, \\
& \quad \text { ACM Transactions on Computational Logic, Vol. V, No. N, June } 2021 .
\end{aligned}
$$


$(\mathfrak{M}, x) \models \forall \varphi$ iff $(\mathfrak{M}, y) \models \varphi$ for all $y \in W$.

Instead of $(\mathfrak{M}, x) \models \varphi$ we write $x \models \varphi$ if $\mathfrak{M}$ is clear from the context.

A formula $\varphi$ is valid in a frame $\mathfrak{F}, \mathfrak{F} \models \varphi$ in symbols, if $\varphi$ is true at every point of every model based on $\mathfrak{F}$. The following facts are well known (see, for example, [Areces et al. 2000]):

FACT 2.1. $\mathrm{K}_{u}$ is the set of formulas that are valid in all frames. $\mathrm{K}_{u}$ is the set of formulas that are valid in all transitive frames. The satisfiability problem is EXPTIME-complete for $\mathrm{K}_{u}$, and PSPACE-complete for $\mathrm{K}_{u}$.

We now formulate the unification problem for normal modal logics with the universal modality.

Definition 2.2. The unification problem for a normal modal $\operatorname{logic} L$ with the universal modality is to decide, given a formula $\varphi$, whether there exists a substitution $s$ such that $s(\varphi) \in L$.

THEOREM 2.3. The unification problem for any normal modal logic between $\mathrm{K}_{u}$ and $\mathrm{K}_{u}$ is undecidable.

The proof proceeds by reduction of some undecidable configuration problem for Minsky machines.

We remind the reader that a Minsky machine (or a register machine with two registers; see, e.g., [Minsky 1961; Ebbinghaus et al. 1994]) is a finite set (program) of instructions for transforming triples $\langle s, m, n\rangle$ of natural numbers, called configurations. The intended meaning of the current configuration $\langle s, m, n\rangle$ is as follows: $s$ is the number (label) of the current machine state and $m, n$ represent the current state of information. Each instruction has one of the four possible forms:

$$
\begin{array}{ll}
s \rightarrow\langle t, 1,0\rangle, & s \rightarrow\langle t,-1,0\rangle\left(\left\langle t^{\prime}, 0,0\right\rangle\right), \\
s \rightarrow\langle t, 0,1\rangle, & s \rightarrow\langle t, 0,-1\rangle\left(\left\langle t^{\prime}, 0,0\right\rangle\right) .
\end{array}
$$

The last of them, for instance, means: transform $\langle s, m, n\rangle$ into $\langle t, m, n-1\rangle$ if $n>0$ and into $\left\langle t^{\prime}, m, n\right\rangle$ if $n=0$. We assume that Minsky machines are deterministic, that is, they can have at most one instruction with a given $s$ in the left-hand side. For a Minsky machine $\boldsymbol{P}$, we write $\boldsymbol{P}:\langle s, m, n\rangle \rightarrow\langle t, k, l\rangle$ if starting with $\langle s, m, n\rangle$ and applying the instructions in $\boldsymbol{P}$, in finitely many steps (possibly, in 0 steps) we can reach $\langle t, k, l\rangle$.

We will use the well known fact (see, e.g., [Chagrov 1990; Chagrov and Zakharyaschev 1997]) that there exist a Minsky program $\boldsymbol{P}$ and a configuration $\mathfrak{a}=\langle s, m, n\rangle$ such that no algorithm can decide, given a configuration $\mathfrak{b}$, whether $\boldsymbol{P}: \mathfrak{a} \rightarrow \mathfrak{b}$.

Fix such a pair $\boldsymbol{P}$ and $\mathfrak{a}=\langle s, m, n\rangle$, and consider the transitive frame $\mathfrak{F}=(W, R)$ shown in Fig. 1, where the points $e(t, k, l)$ represent configurations $\langle t, k, l\rangle$ such that $\boldsymbol{P}:\langle s, m, n\rangle \rightarrow\langle t, k, l\rangle, e(t, k, l)$ 'sees' the points $a_{t}^{0}, a_{k}^{1}, a_{l}^{2}$ representing the components of $\langle t, k, l\rangle$, and $a$ is the only reflexive point of $\mathfrak{F}$. More precisely,

$$
\begin{aligned}
W=\left\{a, b, g, g_{1}, g_{2}, d, d_{1}, d_{2}\right\} \cup\left\{a_{j}^{i} \mid i \leq 2,\right. & j<\omega\} \cup \\
& \{e(t, k, l) \mid \boldsymbol{P}:\langle s, m, n\rangle \rightarrow\langle t, k, l\rangle\}
\end{aligned}
$$

ACM Transactions on Computational Logic, Vol. V, No. N, June 2021. 


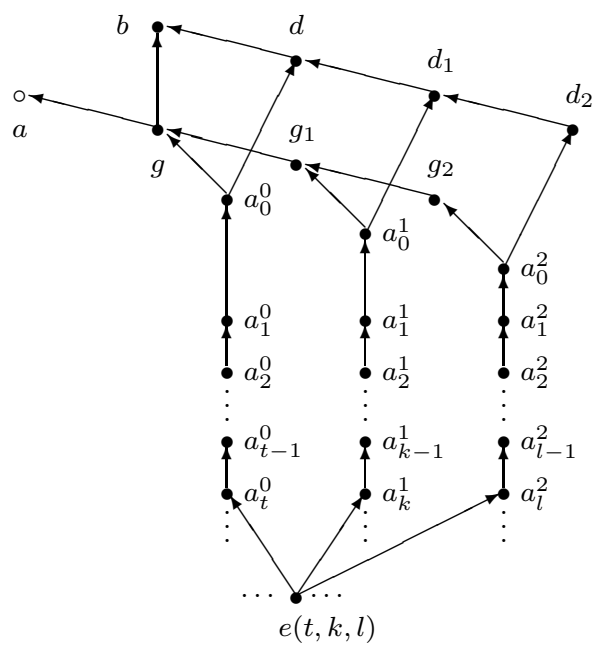

Fig. 1. Frame $\mathfrak{F}$ encoding $\boldsymbol{P}$ and $\mathfrak{a}$.

and $R$ is the transitive closure of the following relation:

$$
\begin{aligned}
& \left\{(a, a),(g, a),(g, b),(d, b),\left(g_{1}, g\right),\left(g_{2}, g_{1}\right),\left(d_{1}, d\right),\left(d_{2}, d_{1}\right)\right. \\
& \left.\left(a_{0}^{0}, g\right),\left(a_{0}^{0}, d\right),\left(a_{0}^{1}, g_{1}\right),\left(a_{0}^{1}, d_{1}\right),\left(a_{0}^{2}, g_{2}\right),\left(a_{0}^{2}, d_{2}\right)\right\} \cup \\
& \quad\left\{\left(a_{j+1}^{i}, a_{j}^{i}\right) \mid i \leq 2, j<\omega\right\} \cup \\
& \quad\left\{\left(e(t, k, l), a_{t}^{0}\right),\left(e(t, k, l), a_{k}^{1}\right),\left(e(t, k, l), a_{l}^{2}\right) \mid e(t, k, l) \in W\right\} .
\end{aligned}
$$

This frame and the formulas below describing it were introduced by A. Chagrov in [Chagrov and Zakharyaschev 1997; Zakharyaschev et al. 2001] where the reader can find further references.

The following variable free formulas characterise the points in $\mathfrak{F}$ in the sense that each of these formulas, denoted by Greek letters with subscripts and/or superscripts, is true in $\mathfrak{F}$ precisely at the point denoted by the corresponding Roman letter with the same subscript and/or superscript (and nowhere else):

$$
\begin{array}{ll}
\alpha=\diamond \top \wedge \square \diamond \top, & \beta=\square \perp, \\
\gamma=\diamond \alpha \wedge \diamond \beta \wedge \neg \diamond^{2} \beta, & \delta=\neg \gamma \wedge \diamond \beta \wedge \neg \diamond^{2} \beta, \\
\delta_{1}=\diamond \delta \wedge \neg \diamond^{2} \delta, & \delta_{2}=\diamond \delta_{1} \wedge \neg \diamond^{2} \delta_{1}, \\
\gamma_{1}=\diamond \gamma \wedge \neg \diamond^{2} \gamma \wedge \neg \diamond \delta, & \gamma_{2}=\diamond \gamma_{1} \wedge \neg \diamond^{2} \gamma_{1} \wedge \neg \diamond \delta, \\
\alpha_{0}^{0}=\diamond \gamma \wedge \diamond \delta \wedge \neg \diamond^{2} \gamma \wedge \neg \diamond^{2} \delta, & \\
\alpha_{0}^{1}=\diamond \gamma_{1} \wedge \diamond \delta_{1} \wedge \neg \diamond^{2} \gamma_{1} \wedge \neg \diamond^{2} \delta_{1}, & \\
\alpha_{0}^{2}=\diamond \gamma_{2} \wedge \diamond \delta_{2} \wedge \neg \diamond^{2} \gamma_{2} \wedge \neg \diamond^{2} \delta_{2}, & \\
\alpha_{j+1}^{i}=\diamond \alpha_{0}^{i} \wedge \diamond \alpha_{j}^{i} \wedge \neg \diamond^{2} \alpha_{j}^{i} \wedge \bigwedge_{i \neq k} \neg \diamond \alpha_{0}^{k},
\end{array}
$$


where $i \in\{0,1,2\}, j \geq 0$. It is worth emphasising that the formulas

$$
\alpha_{j}^{i} \rightarrow \neg \diamond \alpha_{j}^{i} \quad \text { and } \quad \alpha_{j+1}^{i} \rightarrow \diamond \alpha_{0}^{i} \wedge \bigwedge_{k \neq i} \neg \diamond \alpha_{0}^{k}
$$

are valid in all frames for all $i \in\{0,1,2\}, j \geq 0$. We will use this property in what follows.

The formulas characterising the points $e(t, k, l)$ are denoted by $\varepsilon\left(t, \alpha_{k}^{1}, \alpha_{l}^{2}\right)$ and defined as follows, where $\varphi$ and $\psi$ are arbitrary formulas,

$$
\varepsilon(t, \varphi, \psi)=\diamond \alpha_{t}^{0} \wedge \neg \diamond \alpha_{t+1}^{0} \wedge \diamond \varphi \wedge \neg \diamond^{2} \varphi \wedge \diamond \psi \wedge \neg \diamond^{2} \psi .
$$

We also require formulas characterising not only fixed but arbitrary configurations:

$$
\begin{aligned}
\pi_{1} & =\left(\diamond \alpha_{0}^{1} \vee \alpha_{0}^{1}\right) \wedge \neg \diamond \alpha_{0}^{0} \wedge \neg \diamond \alpha_{0}^{2} \wedge p_{1} \wedge \neg \diamond p_{1}, \\
\pi_{2} & =\diamond \alpha_{0}^{1} \wedge \neg \diamond \alpha_{0}^{0} \wedge \neg \diamond \alpha_{0}^{2} \wedge \diamond p_{1} \wedge \neg \diamond^{2} p_{1}, \\
\tau_{1} & =\left(\diamond \alpha_{0}^{2} \vee \alpha_{0}^{2}\right) \wedge \neg \diamond \alpha_{0}^{0} \wedge \neg \diamond \alpha_{0}^{1} \wedge p_{2} \wedge \neg \diamond p_{2}, \\
\tau_{2} & =\diamond \alpha_{0}^{2} \wedge \neg \diamond \alpha_{0}^{0} \wedge \neg \diamond \alpha_{0}^{1} \wedge \diamond p_{2} \wedge \neg \diamond^{2} p_{2} .
\end{aligned}
$$

Observe that in $\mathfrak{F}$, under any valuation, $\pi_{1}$ can be true in at most one point, and this point has to be $a_{j}^{1}$, for some $j \geq 0$. Similarly, $\pi_{2}$ can only we true in at most one point, and this point has to be of the form $a_{j}^{1}$, for some $j>0$. The same applies to $\tau_{1}$ and $\tau_{2}$, but with $a_{j}^{1}$ replaced by $a_{j}^{2}$.

Now we are fully equipped to simulate the behaviour of $\boldsymbol{P}$ on $\mathfrak{a}$ by means of modal formulas with the universal modalities.

With each instruction $I$ in $\boldsymbol{P}$ we associate a formula $A x I$ by taking:

$$
A x I=\exists \varepsilon\left(t, \pi_{1}, \tau_{1}\right) \rightarrow \exists \varepsilon\left(t^{\prime}, \pi_{2}, \tau_{1}\right)
$$

if $I$ is of the form $t \rightarrow\left\langle t^{\prime}, 1,0\right\rangle$,

$$
A x I=\exists \varepsilon\left(t, \pi_{1}, \tau_{1}\right) \rightarrow \exists \varepsilon\left(t^{\prime}, \pi_{1}, \tau_{2}\right)
$$

if $I$ is $t \rightarrow\left\langle t^{\prime}, 0,1\right\rangle$,

$$
A x I=\left(\exists \varepsilon\left(t, \pi_{2}, \tau_{1}\right) \rightarrow \exists \varepsilon\left(t^{\prime}, \pi_{1}, \tau_{1}\right)\right) \wedge\left(\exists \varepsilon\left(t, \alpha_{0}^{1}, \tau_{1}\right) \rightarrow \exists \varepsilon\left(t^{\prime \prime}, \alpha_{0}^{1}, \tau_{1}\right)\right)
$$

if $I$ is $t \rightarrow\left\langle t^{\prime},-1,0\right\rangle\left(\left\langle t^{\prime \prime}, 0,0\right\rangle\right)$, and finally

$$
A x I=\left(\exists \varepsilon\left(t, \pi_{1}, \tau_{2}\right) \rightarrow \exists \varepsilon\left(t^{\prime}, \pi_{1}, \tau_{1}\right)\right) \wedge\left(\exists \varepsilon\left(t, \pi_{1}, \alpha_{0}^{2}\right) \rightarrow \exists \varepsilon\left(t^{\prime \prime}, \pi_{1}, \alpha_{0}^{2}\right)\right)
$$

if $I$ is $t \rightarrow\left\langle t^{\prime}, 0,-1\right\rangle\left(\left\langle t^{\prime \prime}, 0,0\right\rangle\right)$.

The formula simulating $\boldsymbol{P}$ as a whole is

$$
A x P=\bigwedge_{I \in \boldsymbol{P}} A x I .
$$

One can readily check that $\mathfrak{F} \models A x P$.

Now, for each $\mathfrak{b}=\langle t, k, l\rangle$ consider the formula

$$
\psi(\mathfrak{b})=\left(A x P \wedge \exists \varepsilon\left(s, \alpha_{m}^{1}, \alpha_{n}^{2}\right)\right) \rightarrow \exists \varepsilon\left(t, \alpha_{k}^{1}, \alpha_{l}^{2}\right) .
$$

Lemma 2.4. Let $\mathrm{K}_{u} \subseteq L \subseteq \mathrm{K} 4_{u}$. Then $\boldsymbol{P}: \mathfrak{a} \rightarrow \mathfrak{b}$ iff $\psi(\mathfrak{b})$ is unifiable in $L$.

ACM Transactions on Computational Logic, Vol. V, No. N, June 2021. 
Proof of Lemma. $(\Leftarrow)$ Suppose that $\boldsymbol{P}: \mathfrak{a} \not \rightarrow \mathfrak{b}$. Then, by the construction of $\mathfrak{F}$, we have

$$
\mathfrak{F} \models A x P \wedge \exists \varepsilon\left(s, \alpha_{m}^{1}, \alpha_{n}^{2}\right) \quad \text { and } \quad \mathfrak{F} \not \models \exists \varepsilon\left(t, \alpha_{k}^{1}, \alpha_{l}^{2}\right) .
$$

As $\exists \varepsilon\left(t, \alpha_{k}^{1}, \alpha_{l}^{2}\right)$ is variable free, all substitution instances of $\psi(\mathfrak{b})$ are refuted in $\mathfrak{F}$, and so $\psi(\mathfrak{b})$ is not unifiable in any $L \subseteq \mathrm{K} 4_{u}$.

$(\Rightarrow$ ) Conversely, suppose that $\boldsymbol{P}: \mathfrak{a} \rightarrow \mathfrak{b}$. Our aim is to find a substitution $s$ for the variables $p_{1}$ and $p_{2}$ such that $s(\psi(\mathfrak{b})) \in \mathrm{K}_{u}$.

Let

$$
\boldsymbol{P}: \mathfrak{a}=\left\langle t_{0}, k_{0}, l_{0}\right\rangle \stackrel{I_{1}}{\rightarrow}\left\langle t_{1}, k_{1}, l_{1}\right\rangle \stackrel{I_{2}}{\rightarrow} \ldots \stackrel{I_{\ell}}{\rightarrow}\left\langle t_{\ell}, k_{\ell}, l_{\ell}\right\rangle=\mathfrak{b}
$$

be the computation of $\boldsymbol{P}$ starting with $\mathfrak{a}$ and ending with $\mathfrak{b}$, where $I_{j}$ is the instruction from $\boldsymbol{P}$ that is used to transform $\left\langle t_{j-1}, k_{j-1}, l_{j-1}\right\rangle$ into $\left\langle t_{j}, k_{j}, l_{j}\right\rangle$. Consider the formula

$$
\operatorname{defect}_{i}=\exists \varepsilon\left(t_{0}, \alpha_{k_{0}}^{1}, \alpha_{l_{0}}^{2}\right) \wedge \cdots \wedge \exists \varepsilon\left(t_{i}, \alpha_{k_{i}}^{1}, \alpha_{l_{i}}^{2}\right) \wedge \neg \exists \varepsilon\left(t_{i+1}, \alpha_{k_{i+1}}^{1}, \alpha_{l_{i+1}}^{2}\right)
$$

which 'says' that the computation is simulated properly up to the $i$ th step, but there is no point representing the $i+1$ st configuration.

Define the substitution $s$ we need by taking

$$
s\left(p_{1}\right)=\bigvee_{i=0}^{\ell-1} \operatorname{defect}_{i} \wedge \bar{\alpha}_{k_{i}}^{1}, \quad s\left(p_{2}\right)=\bigvee_{i=0}^{\ell-1} \operatorname{defect}_{i} \wedge \bar{\alpha}_{l_{i}}^{2},
$$

where

$$
\bar{\alpha}_{k_{i}}^{1}= \begin{cases}\alpha_{k_{i}}^{1} & \text { if either } k_{i}=0 \text { or } I_{i+1} \neq t_{i} \rightarrow\left\langle t_{i+1},-1,0\right\rangle \\ \alpha_{k_{i}-1}^{1} & \text { if } k_{i} \neq 0 \text { and } I_{i+1}=t_{i} \rightarrow\left\langle t_{i+1},-1,0\right\rangle,\end{cases}
$$

and

$$
\bar{\alpha}_{l_{i}}^{2}= \begin{cases}\alpha_{l_{i}}^{2} & \text { if either } l_{i}=0 \text { or } I_{i+1} \neq t_{i} \rightarrow\left\langle t_{i+1}, 0,-1\right\rangle, \\ \alpha_{l_{i}-1}^{2} & \text { if } l_{i} \neq 0 \text { and } I_{i+1}=t_{i} \rightarrow\left\langle t_{i+1}, 0,-1\right\rangle .\end{cases}
$$

We show now that we have $\mathfrak{G} \models s(\psi(\mathfrak{b}))$ for all frames $\mathfrak{G}$, which clearly means that $\boldsymbol{s}(\psi(\mathfrak{b})) \in \mathrm{K}_{u}$.

Suppose $\mathfrak{G}=(W, R)$ is given. As all formulas considered below, in particular $\boldsymbol{s}(\psi(\mathfrak{b}))$, are variable free, we can write $x \models \psi$ to say that $\psi$ is true at $x$ in some/all models based on $\mathfrak{G}$. Moreover, for any Boolean combination $\psi$ of such formulas starting with $\exists$, we have $x \models \psi$ iff $x^{\prime} \models \psi$ for any $x, x^{\prime} \in W$. Hence, $\mathfrak{G} \not \models \psi$ means that $x \not \models \psi$ for all $x \in W$.

Let us now proceed with the proof. Two cases are possible.

Case 1: $\mathfrak{G} \models \neg \exists \varepsilon\left(t_{0}, \alpha_{k_{0}}^{1}, \alpha_{l_{0}}^{2}\right) \vee \exists \varepsilon\left(t_{\ell}, \alpha_{k_{\ell}}^{1}, \alpha_{l_{\ell}}^{2}\right)$. Then clearly $\mathfrak{G} \models s(\psi(\mathfrak{b}))$.

Case 2: $\mathfrak{G} \models \exists \varepsilon\left(t_{0}, \alpha_{k_{0}}^{1}, \alpha_{l_{0}}^{2}\right) \wedge \neg \exists \varepsilon\left(t_{\ell}, \alpha_{k_{\ell}}^{1}, \alpha_{l_{\ell}}^{2}\right)$. Then there exists some number $i<\ell$ such that $\mathfrak{G} \models \operatorname{defect}_{i}$. It follows that, for all $z \in W$,

$$
z \models s\left(p_{1}\right) \quad \text { iff } \quad z \models \bar{\alpha}_{k_{i}}^{1}, \quad \text { and } \quad z \models s\left(p_{2}\right) \quad \text { iff } \quad z \models \bar{\alpha}_{l_{i}}^{2} .
$$

Claim 2.5. For all $z \in W$, we have (i) $z \models s\left(\pi_{1}\right)$ iff $z \models \bar{\alpha}_{k_{i}}^{1}$, and (ii) $z \models s\left(\tau_{1}\right)$ iff $z \models \bar{\alpha}_{l_{i}}^{2}$. 
Proof of Claim. Suppose $z \in W$ is given. We know that

$$
\boldsymbol{s}\left(\pi_{1}\right)=\left(\diamond \alpha_{0}^{1} \vee \alpha_{0}^{1}\right) \wedge \neg \diamond \alpha_{0}^{0} \wedge \neg \diamond \alpha_{0}^{2} \wedge \boldsymbol{s}\left(p_{1}\right) \wedge \neg \diamond \boldsymbol{s}\left(p_{1}\right) .
$$

Hence, by (4) and (1),

$$
z \models s\left(\pi_{1}\right) \quad \text { iff } \quad z \models\left(\diamond \alpha_{0}^{1} \vee \alpha_{0}^{1}\right) \wedge \neg \diamond \alpha_{0}^{0} \wedge \neg \diamond \alpha_{0}^{2} \wedge \bar{\alpha}_{k_{i}}^{1} \wedge \neg \diamond \bar{\alpha}_{k_{i}}^{1} \quad \text { iff } \quad z \models \bar{\alpha}_{k_{i}}^{1} .
$$

(ii) is considered analogously.

Claim 2.6. For all $z \in W$, (i) $z \models s\left(\pi_{2}\right)$ iff $z \models \bar{\alpha}_{k_{i}+1}^{1}$, and (ii) $z \models s\left(\tau_{2}\right)$ iff $z \models \bar{\alpha}_{l_{i}+1}^{2}$.

Proof of Claim. Suppose $z \in W$ is given. We know that

$$
\boldsymbol{s}\left(\pi_{2}\right)=\diamond \alpha_{0}^{1} \wedge \neg \diamond \alpha_{0}^{0} \wedge \neg \diamond \alpha_{0}^{2} \wedge \diamond \boldsymbol{s}\left(p_{1}\right) \wedge \neg \diamond^{2} \boldsymbol{s}\left(p_{1}\right) .
$$

Hence, by (4),

$$
z \models s\left(\pi_{2}\right) \quad \text { iff } \quad z \models \diamond \alpha_{0}^{1} \wedge \neg \diamond \alpha_{0}^{0} \wedge \neg \diamond \alpha_{0}^{2} \wedge \diamond \bar{\alpha}_{k_{i}}^{1} \wedge \neg \diamond^{2} \bar{\alpha}_{k_{i}}^{1} .
$$

But, according to (1), the latter formula is equivalent to the definition of $\bar{\alpha}_{k_{i}+1}^{1}$, which proves the claim.

We now make a case distinction according to rule $I_{i+1}$ used to transform $\left\langle t_{i}, k_{i}, l_{i}\right\rangle$ to $\left\langle t_{i+1}, k_{i+1}, l_{i+1}\right\rangle$.

Case 1: $I_{i+1}=t_{i} \rightarrow\left\langle t_{i+1}, 1,0\right\rangle$. Our aim is to show that

(a) $\mathfrak{G} \models s\left(\exists \varepsilon\left(t_{i}, \pi_{1}, \tau_{1}\right)\right)$ and

(b) $\mathfrak{G} \not \models \boldsymbol{s}\left(\exists \varepsilon\left(t_{i+1}, \pi_{2}, \tau_{1}\right)\right)$,

for then we would have $\mathfrak{G} \not \models s(A x P)$, and so $\mathfrak{G} \models s(\psi(\mathfrak{b}))$.

(a) As $\mathfrak{G} \models \exists \varepsilon\left(t_{i}, \alpha_{k_{i}}^{1}, \alpha_{l_{i}}^{2}\right)$, we have some $z \in W$ such that

$$
z \models \diamond \alpha_{t_{i}}^{0} \wedge \neg \diamond \alpha_{t_{i}+1}^{0} \wedge \diamond \alpha_{k_{i}}^{1} \wedge \neg \diamond^{2} \alpha_{k_{i}}^{1} \wedge \diamond \alpha_{l_{i}}^{2} \wedge \neg \diamond^{2} \alpha_{l_{i}}^{2} .
$$

By Claim 2.5, we then have

$$
z \models \diamond \alpha_{t_{i}}^{0} \wedge \neg \diamond \alpha_{t_{i}+1}^{0} \wedge \diamond \boldsymbol{s}\left(\pi_{1}\right) \wedge \neg \diamond^{2} \boldsymbol{s}\left(\pi_{1}\right) \wedge \diamond \boldsymbol{s}\left(\tau_{1}\right) \wedge \neg \diamond^{2} \boldsymbol{s}\left(\tau_{1}\right),
$$

which means that $z \models s\left(\varepsilon\left(t_{i}, \pi_{1}, \tau_{1}\right)\right)$, and so $\mathfrak{G} \models s\left(\exists \varepsilon\left(t_{i}, \pi_{1}, \tau_{1}\right)\right)$.

(b) Suppose that $\mathfrak{G} \not \models s\left(\exists \varepsilon\left(t_{i+1}, \pi_{2}, \tau_{1}\right)\right)$ does not hold. Then there is $x \in W$ with

$$
x \models \varepsilon\left(t_{i+1}, s\left(\pi_{2}\right), s\left(\tau_{1}\right)\right),
$$

that is,

$$
x \models \diamond \alpha_{t_{i+1}}^{0} \wedge \neg \diamond \alpha_{t_{i+1}+1}^{0} \wedge \diamond \boldsymbol{s}\left(\pi_{2}\right) \wedge \neg \diamond^{2} \boldsymbol{s}\left(\pi_{2}\right) \wedge \diamond \boldsymbol{s}\left(\tau_{1}\right) \wedge \neg \diamond^{2} \boldsymbol{s}\left(\tau_{1}\right) .
$$

By Claims 2.5 and 2.6, we then have

$$
x \models \diamond \alpha_{t_{i+1}}^{0} \wedge \neg \diamond \alpha_{t_{i+1}+1}^{0} \wedge \diamond \alpha_{k_{i}+1}^{1} \wedge \neg \diamond^{2} \alpha_{k_{i}+1}^{1} \wedge \diamond \alpha_{l_{i}}^{2} \wedge \neg \diamond^{2} \alpha_{l_{i}}^{2}
$$

which means

$$
x \models \varepsilon\left(t_{i+1}, \alpha_{k_{i}+1}^{1}, \alpha_{l_{i}}^{2}\right) .
$$

ACM Transactions on Computational Logic, Vol. V, No. N, June 2021. 
Now recall that $\alpha_{k_{i}+1}^{1}=\alpha_{k_{i+1}}^{1}$ and $\alpha_{l_{i}}=\alpha_{l_{i+1}}$, that is, we have

$$
x \models \varepsilon\left(t_{i+1}, \alpha_{k_{i+1}}^{1}, \alpha_{l_{i+1}}^{2}\right),
$$

and so $\mathfrak{G} \models \exists \varepsilon\left(t_{i+1}, \alpha_{k_{i+1}}^{1}, \alpha_{l_{i+1}}^{2}\right)$, contrary to $\mathfrak{G} \models \operatorname{defect}_{i}$.

Case 2: $I_{i+1}$ is of the form $t_{i} \rightarrow\left\langle t_{i+1}^{\prime},-1,0\right\rangle\left(\left\langle t_{i+1}^{\prime \prime}, 0,0\right\rangle\right)$. Suppose first that $k_{i}=0$, that is, the actual instruction is $I_{i+1}=t_{i} \rightarrow\left\langle t_{i+1}, 0,0\right\rangle$. We need to show that

(a) $\mathfrak{G} \models \boldsymbol{s}\left(\exists \varepsilon\left(t_{i}, \alpha_{0}^{1}, \tau_{1}\right)\right)$ and

(b) $\mathfrak{G} \not \models \boldsymbol{s}\left(\exists \varepsilon\left(t_{i+1}, \alpha_{0}^{1}, \tau_{1}\right)\right)$,

which, as before, would imply $\mathfrak{G} \models s(\psi(\mathfrak{b}))$.

(a) As $\mathfrak{G} \models \exists \varepsilon\left(t_{i}, \alpha_{0}^{1}, \alpha_{l_{i}}^{2}\right)$, we have $x \in W$ such that

$$
x \models \diamond \alpha_{t_{i}}^{0} \wedge \neg \diamond \alpha_{t_{i}+1}^{0} \wedge \diamond \alpha_{0}^{1} \wedge \neg \diamond^{2} \alpha_{0}^{1} \wedge \diamond \alpha_{l_{i}}^{2} \wedge \neg \diamond^{2} \alpha_{l_{i}}^{2},
$$

from which, by Claim 2.5,

$$
x \models \diamond \alpha_{t_{i}}^{0} \wedge \neg \diamond \alpha_{t_{i}+1}^{0} \wedge \diamond \alpha_{0}^{1} \wedge \neg \diamond^{2} \alpha_{0}^{1} \wedge \diamond \boldsymbol{s}\left(\tau_{1}\right) \wedge \neg \diamond^{2} \boldsymbol{s}\left(\tau_{1}\right) .
$$

Thus we have $x \models \exists \varepsilon\left(t_{i}, \alpha_{0}^{1}, \tau_{1}\right)$. (b) is proved similarly and left to the reader.

Suppose now that $k_{i}>0$, that is, the instruction $I_{i+1}=t_{i} \rightarrow\left\langle t_{i+1},-1,0\right\rangle$ was actually used. This time we need to show that

(a) $\mathfrak{G} \models s\left(\exists \varepsilon\left(t_{i}, \pi_{2}, \tau_{1}\right)\right)$ and

(b) $\mathfrak{G} \not \models \boldsymbol{s}\left(\exists \varepsilon\left(t_{i+1}, \pi_{1}, \tau_{1}\right)\right)$.

(a) Since $\mathfrak{G} \models \exists \varepsilon\left(t_{i}, \alpha_{k_{i}}^{1}, \alpha_{l_{i}}^{2}\right)$, we have $x \in W$ such that

$$
x \models \diamond \alpha_{t_{i}}^{0} \wedge \neg \diamond \alpha_{t_{i}+1}^{0} \wedge \diamond \alpha_{k_{i}}^{1} \wedge \neg \diamond^{2} \alpha_{k_{i}}^{1} \wedge \diamond \alpha_{l_{i}}^{2} \wedge \neg \diamond^{2} \alpha_{l_{i}}^{2} .
$$

Clearly, it is sufficient to show that

$$
x \models \diamond \alpha_{t_{i}}^{0} \wedge \neg \diamond \alpha_{t_{i}+1}^{0} \wedge \diamond \boldsymbol{s}\left(\pi_{2}\right) \wedge \neg \diamond^{2} \boldsymbol{s}\left(\pi_{2}\right) \wedge \diamond \boldsymbol{s}\left(\tau_{1}\right) \wedge \neg \diamond^{2} \boldsymbol{s}\left(\tau_{1}\right) .
$$

Observe that in this case $\bar{\alpha}_{k_{i}}^{1}=\alpha_{k_{i}-1}$. Hence, by Claim 2.6, for all $z \in W$ we have $z \models s\left(\pi_{2}\right)$ iff $z \models \alpha_{k_{i}}^{1}$. So it remains to use Claims 2.5 and 2.6.

(b) Suppose otherwise, that is, $\mathfrak{G} \models \boldsymbol{s}\left(\exists \varepsilon\left(t_{i+1}, \pi_{1}, \tau_{1}\right)\right)$. Then there exists $x \in W$ such that

$$
x \models \diamond \alpha_{t_{i+1}}^{0} \wedge \neg \diamond \alpha_{t_{i+1}+1}^{0} \wedge \diamond \boldsymbol{s}\left(\pi_{1}\right) \wedge \neg \diamond^{2} \boldsymbol{s}\left(\pi_{1}\right) \wedge \diamond \boldsymbol{s}\left(\tau_{1}\right) \wedge \neg \diamond^{2} \boldsymbol{s}\left(\tau_{1}\right) .
$$

By Claim 2.5, this implies

$$
x \models \diamond \alpha_{t_{i+1}}^{0} \wedge \neg \diamond \alpha_{t_{i+1}+1}^{0} \wedge \diamond \alpha_{k_{i}-1}^{1} \wedge \neg \diamond^{2} \alpha_{k_{i}-1}^{1} \wedge \diamond \alpha_{l_{i}}^{2} \wedge \neg \diamond^{2} \alpha_{l_{i}}^{2},
$$

that is,

$$
x \models \varepsilon\left(t_{i+1}, \alpha_{k_{i}-1}^{1}, \alpha_{l_{i}}^{2}\right)
$$

which leads to a contradiction, because $\alpha_{k_{i}-1}^{1}=\alpha_{k_{i+1}}^{1}$ and $\alpha_{l_{i}}=\alpha_{l_{i+1}}$, and therefore we must have $\mathfrak{G} \models \exists \varepsilon\left(t_{i+1}, \alpha_{k_{i+1}}^{1}, \alpha_{l_{i+1}}^{2}\right)$.

The remaining two types of instructions (where the third component changes) are dual to the ones considered above. We leave these cases to the reader. 
This completes the proof of Lemma 2.4. Theorem 2.3 follows immediately in view of the choice of $\boldsymbol{P}$ and $\alpha$.

Observe that Theorem 2.3 can be proved for multimodal $\mathrm{K}_{u}$ and $\mathrm{K} 4_{u}$ as well. In this case, in the frame $\mathfrak{F}$ considered above, the additional operators can be interpreted by the empty relation. By a proper modification of the frame $\mathfrak{F}$ in Fig. 1, this theorem can also be extended to some logics above $\mathrm{K} 4_{u}$, for example, $\mathrm{GL}_{u}$.

Definition 2.7. The admissibility problem for inference rules for a normal modal $\operatorname{logic} L$ with the universal modality is to decide, given an inference rule $\varphi_{1}, \ldots, \varphi_{n} / \varphi$, whether $s\left(\varphi_{1}\right) \in L, \ldots, s\left(\varphi_{n}\right) \in L$ imply $s(\varphi) \in L$, for every substitution $s$.

As an immediate consequence of Theorem 2.3 we obtain the following:

THEOREM 2.8. The admissibility problem for any normal modal logic $L$ between $\mathrm{K}_{u}$ and $\mathrm{K}_{u}$ is undecidable.

Minor modifications of the proof above can be used to prove undecidability of the unification and admissibility problems for various modal logics in which the universal modality is definable. An interesting example is PDL with converse, i.e., the extension of propositional dynamic logic with the converse constructor on programs: if $\alpha$ is a program, then $\alpha^{-1}$ is a program which is interpreted by the converse of the relation interpreting $\alpha$. (We do not provide detailed definitions of the syntax and semantics here but refer the reader to [Harel et al. 2000].) The undecidability proof for the unification problem (for substitutions instead of propositional variables rather than atomic programs!) is carried out by taking an atomic program $\alpha$ and replacing, in the proof above, the operator $\square$ with $[\alpha]$ and the universal modality $\forall$ with $\left[\left(\alpha \cup \alpha^{-1}\right)^{*}\right]$.

It seems worth mentioning, however, that the unification problem is trivially decidable for any normal modal $\operatorname{logic} L$ with $\neg \square \perp \in L$. To see this, recall that a substitution $s$ is called ground if it replaces each propositional variable by a variable free formula (that is, a formula constructed from $\perp$ and $T$ only). Obviously, it is always the case that if there exists a substitution $s$ such that $s(\varphi) \in L$, then there exists a ground substitution $s^{\prime}$ with $s^{\prime}(\varphi) \in L$. But if $\neg \square \perp \in L$, then there are, up to equivalence in $L$, only two different variable free formulas, namely, $\perp$ and $T$. Thus, to decide whether a formula $\varphi$ is unifiable in $L$ it is sufficient to check whether any of the ground substitutions makes $\varphi$ equivalent to $T$ (which can be done in Boolean logic). A well known example of such a logic is $\mathbf{S} 4_{u}$, S4 with the universal modality. Note that the admissibility problem for $\mathrm{S} 4_{u}$ might nevertheless be undecidable. We leave this as an interesting open problem.

\section{UNIFICATION MODULO EQUATIONAL THEORIES}

The results presented above can be reformulated as undecidability results for the well-known notion of unification modulo equational theories [Baader and Siekmann 1994; Baader and Snyder 2001].

Consider the equational theory $\mathrm{BAO}_{2}$ of Boolean algebras with operators $\square_{1}$ and $\square_{2}$, which consists of an axiomatisation BA of the variety of Boolean algebras (say, in the signature with the binary connective $\wedge$, unary connective $\neg$ and constant 1 )

ACM Transactions on Computational Logic, Vol. V, No. N, June 2021. 
together with the equations

$$
\square_{i}(x \wedge y)=\square_{i} x \wedge \square_{i} y \quad \text { and } \quad \square_{i} 1=1,
$$

for $i=1,2$. Let $T$ be any set of equations over the signature of Boolean algebras with two operators. Then the unification problem modulo $\mathrm{BAO}_{2} \cup T$ is to decide, given an equation $t_{1}=t_{2}$ over the signature of $\mathrm{BAO}_{2}$, whether there exists a substitution $s$ such that

$$
\boldsymbol{s}\left(t_{1}\right)=\mathrm{BAO}_{2} \cup T \quad \boldsymbol{s}\left(t_{2}\right),
$$

that is, whether there exists a substitution $s$ such that the equation $s\left(t_{1}\right)=s\left(t_{2}\right)$ is valid in all algebras where the equations in $\mathrm{BAO}_{2} \cup T$ hold true. For a term $t$, let $t^{p}$ denote the propositional modal formula that is obtained from $t$ by replacing its (individual) variables with (mutually distinct) propositional variables. We may assume that.$^{p}$ is a bijection between the terms $t$ over the signature of $\mathrm{BAO}_{2}$ and the modal formulas with modal operators $\square_{1}$ and $\square_{2}$. Denote by $\cdot^{-p}$ the inverse of this function. It is well-known (see, e.g., [Venema 2006]) that a modal formula $\varphi$ is valid in the smallest normal modal logic $L$ containing the formulas

$$
\left\{t_{1}^{p} \leftrightarrow t_{2}^{p} \mid t_{1}=t_{2} \in T\right\}
$$

if, and only if, $\varphi^{-p}$ is valid in all algebras validating $\mathrm{BAO}_{2} \cup T$. The appropriate converse statement is also easily formulated. It follows that the unification problem modulo $\mathrm{BAO}_{2} \cup T$ is decidable if, and only if, the unification problem for $L$ is decidable. Clearly, it remains an open question whether the unification problem modulo $\mathrm{BAO}_{2}$ is decidable. However, if $T$ consists of the following inequalities (saying that $\square_{1}$ is the universal box)

$$
\square_{1} x \leq \square_{2} x, \quad \square_{1} x \leq x, \quad \square_{1} x \leq \square_{1} \square_{1} x, \quad x \leq \square_{1} \neg \square_{1} \neg x,
$$

then Theorem 2.3 implies that the unification problem modulo $\mathrm{BAO}_{2} \cup T$ is undecidable.

\section{UNIFICATION IN MODAL LOGICS WITH NOMINALS}

Let us now consider the extension of the language $\mathcal{L}$ with nominals. More precisely, denote by $\mathcal{H}_{2}$ the propositional language constructed from

—an infinite list $p_{1}, p_{2}, \ldots$ of propositional variables and

—an infinite list $n_{1}, n_{2}, \ldots$ of nominals

using the standard Boolean connectives and two modal operators $\square$ and $\square_{h}$ (instead of $\square$ and $\forall$ in $\mathcal{L}) .{ }^{1} \mathcal{H}_{2}$-formulas are interpreted in frames of the form $\mathfrak{F}=(W, R, S)$ where $R, S \subseteq W \times W$. As before, a model is a pair $\mathfrak{M}=(\mathfrak{F}, \mathfrak{V})$, where $\mathfrak{V}$ is a valuation function that assigns to each $p_{i}$ a subset $\mathfrak{V}\left(p_{i}\right)$ of $W$ and to each $n_{i}$ a singleton subset $\mathfrak{V}\left(n_{i}\right)$ of $W$. The truth-relation, $(\mathfrak{M}, x) \models \varphi$, is defined as above with two extra clauses:

$$
(\mathfrak{M}, x) \models n_{i} \text { iff }\{x\}=\mathfrak{V}\left(n_{i}\right),
$$

\footnotetext{
${ }^{1}$ The language with infinitely many modal operators and nominals is often denoted by $\mathcal{H}$ and called the minimal hybrid logic; see, e.g., [Areces and ten Cate 2006].
} 
$(\mathfrak{M}, x) \models \square_{h} \psi$ iff $(\mathfrak{M}, y) \models \psi$ for all $y \in W$ with $x S y$.

Denote by $\mathrm{K}_{\mathcal{H}_{2}}$ the set of all $\mathcal{H}_{2}$-formulas that are valid in all frames, and denote by $\mathrm{K}_{\mathcal{H}_{2}} \oplus 45$ the set of $\mathcal{H}_{2}$-formulas that are valid in all frames $(W, R, S)$ with transitive $R$ and $S=W \times W$. A proof of the following result can be found in [Areces et al. 2000]:

FACT 4.1. The satisfiability problem for $\mathrm{K}_{\mathcal{H}_{2}}$ is PSPACE-complete, while for $\mathrm{K}_{\mathcal{H}_{2}} \oplus 45$ it is EXPTIME-complete.

A substitution $s$ for $\mathcal{H}_{2}$ is a map from the set of propositional variables into $\mathcal{H}_{2}$. In particular, any substitution leaves nominals intact. ${ }^{2}$ The unification and admissibility problems for modal logics with nominals are formulated in exactly the same way as before.

THEOREM 4.2. The unification problem and, therefore, the admissibility problem for any logic $L$ between $\mathrm{K}_{\mathcal{H}_{2}}$ and $\mathrm{K}_{\mathcal{H}_{2}} \oplus 45$ are undecidable.

The proof of this theorem is similar to the proof of Theorem 2.3. Here we only show how to modify the encoding of Minsky machine computations from Section 2. The main difference is that now the language does not contain the universal modality which can refer to all points in the frame in order to say, e.g., that a certain configuration is (not) reachable. To overcome this problem, we will use one nominal, let us call it $n$, which, if accessible from a point $x$ (via $R$ and $S$ ), will be forced to be accessible from all points located within a certain distance from $x$. This trick will provide us with a 'surrogate' universal modality which behaves, locally, similarly to the standard one.

From now on we will be using the following abbreviation, where $\varphi$ is an $\mathcal{H}_{2^{-}}$formula:

$$
\exists \varphi=\diamond_{h}\left(n \wedge \diamond_{h} \varphi\right) .
$$

The defined operator $\exists$ will play the role of our surrogate universal diamond.

Consider again a Minsky program $\boldsymbol{P}$ and a configuration $\mathfrak{a}=\langle s, m, n\rangle$ such that it is undecidable, given a configuration $\mathfrak{b}$, whether $\boldsymbol{P}: \mathfrak{a} \rightarrow \mathfrak{b}$. The frame $\mathfrak{F}=(W, R, S)$ encoding $\mathfrak{F}$ and $\mathfrak{a}$ is defined as in Fig. 1 , with $S=W \times W$. For each instruction $I$, we introduce the formula $A x I$ in precisely the same way as before, with $\exists$ defined by (5).

The first important difference between the two constructions is the definition of $A x P$. Let $N o m$ denote the conjunction of all $\mathcal{H}_{2}$-formulas of the form

$$
\diamond_{h} n \rightarrow M \diamond_{h} n \text { and } M^{\prime} \diamond_{h} n \rightarrow \diamond_{h} n
$$

where $M$ is any sequence of $\square$ and $\square_{h}$ of length $\leq 6$, and $M^{\prime}$ is any sequence of $\diamond$ and $\diamond_{h}$ of length $\leq 6$. To explain the meaning of $N o m$, consider a model $(\mathfrak{G}, \mathfrak{V})$ based on some frame $\mathfrak{G}=(W, R, S)$. Let $x_{0} \in W$. We say that $x \in W$ is of distance $\leq m$ from $x_{0}$ if there exists a sequence

$$
x_{0} S^{\prime} x_{1} S^{\prime} x_{2} \cdots x_{k-1} S^{\prime} x_{k}=x,
$$

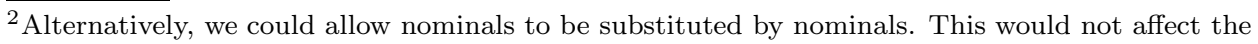
undecidability result.
}

ACM Transactions on Computational Logic, Vol. V, No. N, June 2021. 
where $S^{\prime}=R \cup S$ and $k \leq m$. Now assume that $x_{0} \models N o m$. Then either all points of distance $\leq 6$ from $x_{0}$ 'see' $\mathfrak{V}(n)$ via $S$, or no point of distance $\leq 6$ from $x_{0}$ sees $\mathfrak{V}(n)$ via $S$. In particular, $x_{0} \models \exists \varphi$ if, and only if, $x \models \exists \varphi$ for all $x$ of distance $\leq 6$ from $x_{0}$, and $x_{0} \not \models \exists \varphi$ if, and only if, $x \not \models \exists \varphi$ for all $x$ of distance $\leq 6$ from $x_{0}$.

The formula simulating $\boldsymbol{P}$ as a whole in this case is

$$
A x P=\bigwedge_{I \in \boldsymbol{P}} A x I \wedge \text { Nom. }
$$

Consider the frame $\mathfrak{F}=(W, R, S)$ in Fig. 1 (with $S=W \times W$ ). Then, no matter which singleton set interprets $n$, the new operator $\exists$ is always interpreted by the universal relation. Hence, as before we have $\mathfrak{F} \models A x P$.

Now, for each $\mathfrak{b}=\langle t, k, l\rangle$ consider (as before) the formula

$$
\psi(\mathfrak{b})=A x P \wedge \exists \varepsilon\left(s, \alpha_{m}^{1}, \alpha_{n}^{2}\right) \rightarrow \exists \varepsilon\left(t, \alpha_{k}^{1}, \alpha_{l}^{2}\right) .
$$

Lemma 4.3. $\boldsymbol{P}: \mathfrak{a} \rightarrow \mathfrak{b}$ iff $\psi(\mathfrak{b})$ is unifiable in $L$, where $\mathrm{K}_{\mathcal{H}_{2}} \subseteq L \subseteq \mathrm{K}_{\mathcal{H}_{2}} \oplus 45$.

Proof of Lemma. The proof of $(\Leftarrow)$ is exactly as before.

$(\Rightarrow)$ Suppose that $\boldsymbol{P}: \mathfrak{a} \rightarrow \mathfrak{b}$. Our aim is to find a substitution $s$ for the variables $p_{1}$ and $p_{2}$ such that $\boldsymbol{s}(\psi(\mathfrak{b})) \in \mathrm{K}_{\mathcal{H}_{2}}$. The definition of the substitution is as before. Let

$$
\boldsymbol{P}: \mathfrak{a}=\left\langle t_{0}, k_{0}, l_{0}\right\rangle \stackrel{I_{1}}{\rightarrow}\left\langle t_{1}, k_{1}, l_{1}\right\rangle \stackrel{I_{2}}{\rightarrow} \ldots \stackrel{I_{\ell}}{\rightarrow}\left\langle t_{\ell}, k_{\ell}, l_{\ell}\right\rangle=\mathfrak{b}
$$

be the computation of $\boldsymbol{P}$ starting with $\mathfrak{a}$ and ending with $\mathfrak{b}$. Then we define $s$ by means of (3), where defect $_{i}$ is given by (2).

We have to show that, for all frames $\mathfrak{G}$, we have $\mathfrak{G} \models s(\psi(\mathfrak{b}))$. Note that now we cannot assume that $\exists$ is interpreted by the universal relation.

Suppose that we are given a frame $\mathfrak{G}=(W, R, S)$, a valuation $\mathfrak{V}$ in it, and some $x_{0} \in W$. We write $\left\{n^{\mathfrak{V}}\right\}$ for $\mathfrak{V}(n)$, and $x \models \psi$ for $(\mathfrak{G}, \mathfrak{V}, x) \models \psi$. As before, two cases are possible.

Case 1: $x_{0} \models \neg \exists \varepsilon\left(t_{0}, \alpha_{k_{0}}^{1}, \alpha_{l_{0}}^{2}\right) \vee \exists \varepsilon\left(t_{\ell}, \alpha_{k_{\ell}}^{1}, \alpha_{l_{\ell}}^{2}\right)$. Then clearly $x_{0} \models s(\psi(\mathfrak{b}))$.

Case 2: $x_{0} \models \exists \varepsilon\left(t_{0}, \alpha_{k_{0}}^{1}, \alpha_{l_{0}}^{2}\right) \wedge \neg \exists \varepsilon\left(t_{\ell}, \alpha_{k_{\ell}}^{1}, \alpha_{l_{\ell}}^{2}\right)$. If $x_{0} \not \models s(N o m)$ then obviously $x_{0} \models s(\psi(\mathfrak{b}))$, and we are done. So assume that $x_{0} \models s(N o m)$. Then there exists some number $i<\ell$ such that $x_{0} \models$ defect $_{i}$.

Claim 4.4. For all points $x$ of distance $\leq 6$ from $x_{0}, x \models$ defect $_{i}$. So, for all such $x$, we have $x \models s\left(p_{1}\right)$ iff $x \models \bar{\alpha}_{k_{i}}^{1}$, and $x \models s\left(p_{2}\right)$ iff $x \models \bar{\alpha}_{l_{i}}^{2}$.

Proof of Claim. Follows immediately from $x_{0} \models$ Nom.

Claim 4.5. For all $x$ of distance $\leq 5$ from $x_{0}$, we have (i) $x \models s\left(\pi_{1}\right)$ iff $x \models \bar{\alpha}_{k_{i}}^{1}$, and (ii) $x \models s\left(\tau_{1}\right)$ iff $x \models \alpha_{l_{i}}^{2}$.

Proof of Claim. We only prove (i). Suppose $x$ is given. We know that

$$
\boldsymbol{s}\left(\pi_{1}\right)=\left(\diamond \alpha_{0}^{1} \vee \alpha_{0}^{1}\right) \wedge \neg \diamond \alpha_{0}^{0} \wedge \neg \diamond \alpha_{0}^{2} \wedge \boldsymbol{s}\left(p_{1}\right) \wedge \neg \diamond \boldsymbol{s}\left(p_{1}\right) .
$$

Hence, by Claim 4.4,

$$
x \models s\left(\pi_{1}\right) \quad \text { iff } \quad x \models\left(\diamond \alpha_{0}^{1} \vee \alpha_{0}^{1}\right) \wedge \neg \diamond \alpha_{0}^{0} \wedge \neg \diamond \alpha_{0}^{2} \wedge \bar{\alpha}_{k_{i}}^{1} \wedge \neg \diamond \bar{\alpha}_{k_{i}}^{1} .
$$

(Observe that $s\left(p_{1}\right)$ occurs within the scope of a $\diamond$. Hence, we obtain this equivalence only for points of distance $\leq 5$ from $x_{0}$.) But this is equivalent to $x \models \bar{\alpha}_{k_{i}}^{1}$. 
Claim 4.6. For all $x$ of distance $\leq 4$ from $x_{0}$, (i) $x \models s\left(\pi_{2}\right)$ iff $z \models \bar{\alpha}_{k_{i}+1}^{1}$, and (ii) $x \models s\left(\tau_{2}\right)$ iff $z \models \bar{\alpha}_{l_{i}+1}^{2}$.

Proof of Claim. We only prove (i). Suppose $x$ is given. We know that

$$
\boldsymbol{s}\left(\pi_{2}\right)=\diamond \alpha_{0}^{1} \wedge \neg \diamond \alpha_{0}^{0} \wedge \neg \diamond \alpha_{0}^{2} \wedge \diamond \boldsymbol{s}\left(p_{1}\right) \wedge \neg \diamond^{2} \boldsymbol{s}\left(p_{1}\right) .
$$

Hence, by Claim 4.4,

$$
x \models s\left(\pi_{2}\right) \quad \text { iff } \quad x \models \diamond \alpha_{0}^{1} \wedge \neg \diamond \alpha_{0}^{0} \wedge \neg \diamond \alpha_{0}^{2} \wedge \diamond \bar{\alpha}_{k_{i}}^{1} \wedge \neg \diamond^{2} \bar{\alpha}_{k_{i}}^{1} .
$$

(In this case $\boldsymbol{s}\left(p_{1}\right)$ occurs within the scope of a $\diamond^{2}$. Therefore, we obtain this equivalence for points $x$ of distance $\leq 4$ from $x_{0}$.) But this formula is in fact the definition of $\bar{\alpha}_{k_{i}+1}^{1}$.

As in the proof of Lemma 2.4, we now make a case distinction according to rule $I_{i+1}$ used to transform $\left\langle t_{i}, k_{i}, l_{i}\right\rangle$ to $\left\langle t_{i+1}, k_{i+1}, l_{i+1}\right\rangle$. Here we only consider the case of $I_{i+1}=t_{i} \rightarrow\left\langle t_{i+1}, 1,0\right\rangle$, and leave the remaining three cases to the reader. We need to show that

(a) $x_{0} \models s\left(\exists \varepsilon\left(t_{i}, \pi_{1}, \tau_{1}\right)\right)$ and

(b) $x_{0} \not \models s\left(\exists \varepsilon\left(t_{i+1}, \pi_{2}, \tau_{1}\right)\right)$,

which, as before, would imply $x_{0} \models s(\psi(\mathfrak{b}))$.

(a) As $x_{0} \models \exists \varepsilon\left(t_{i}, \alpha_{k_{i}}^{1}, \alpha_{l_{i}}^{2}\right)$, we have some $z$ such that $x_{0} S n^{\mathfrak{V}} S z$ and

$$
z \models \diamond \alpha_{t_{i}}^{0} \wedge \neg \diamond \alpha_{t_{i}+1}^{0} \wedge \diamond \alpha_{k_{i}}^{1} \wedge \neg \diamond^{2} \alpha_{k_{i}}^{1} \wedge \diamond \alpha_{l_{i}}^{2} \wedge \neg \diamond^{2} \alpha_{l_{i}}^{2} .
$$

Clearly, it is sufficient to show

$$
z \models \diamond \alpha_{t_{i}}^{0} \wedge \neg \diamond \alpha_{t_{i}+1}^{0} \wedge \diamond \boldsymbol{s}\left(\pi_{1}\right) \wedge \neg \diamond^{2} \boldsymbol{s}\left(\pi_{1}\right) \wedge \diamond \boldsymbol{s}\left(\tau_{1}\right) \wedge \neg \diamond^{2} \boldsymbol{s}\left(\tau_{1}\right) .
$$

But this follows from Claim 4.5: just observe that $z$ is of distance $\leq 2$ from $x_{0}$, while $\boldsymbol{s}\left(\pi_{1}\right)$ and $\boldsymbol{s}\left(\tau_{1}\right)$ occur within the scope of $\diamond^{2}$.

(b) To show $x_{0} \not \forall s\left(\exists \varepsilon\left(t_{i+1}, \pi_{2}, \tau_{1}\right)\right)$, suppose otherwise. Then there is $z$ such that $x_{0} S n^{\mathfrak{V}} S z$ and

$$
z \models \varepsilon\left(t_{i+1}, s\left(\pi_{2}\right), s\left(\tau_{1}\right)\right) .
$$

This means that

$$
z \models \diamond \alpha_{t_{i+1}}^{0} \wedge \neg \diamond \alpha_{t_{i+1}+1}^{0} \wedge \diamond \boldsymbol{s}\left(\pi_{2}\right) \wedge \neg \diamond^{2} \boldsymbol{s}\left(\pi_{2}\right) \wedge \diamond \boldsymbol{s}\left(\tau_{1}\right) \wedge \neg \diamond^{2} \boldsymbol{s}\left(\tau_{1}\right) .
$$

By Claims 4.5 and 4.6 this implies

$$
z \models \diamond \alpha_{t_{i+1}}^{0} \wedge \neg \diamond \alpha_{t_{i+1}+1}^{0} \wedge \diamond \alpha_{k_{i}+1}^{1} \wedge \neg \diamond^{2} \alpha_{k_{i}+1}^{1} \wedge \diamond \alpha_{l_{i}}^{2} \wedge \neg \diamond^{2} \alpha_{l_{i}}^{2} .
$$

It follows that

$$
z \models \varepsilon\left(t_{i+1}, \alpha_{k_{i}+1}^{1}, \alpha_{l_{i}}^{2}\right)
$$

and we arrive at a contradiction, because $\alpha_{k_{i}+1}^{1}=\alpha_{k_{i+1}}^{1}$.

This completes the proofs of Lemma 4.3 and Theorem 4.2.

ACM Transactions on Computational Logic, Vol. V, No. N, June 2021. 


\section{APPLICATIONS TO DESCRIPTION LOGICS}

In this section, we briefly comment on the consequences of our results in the context of description logics [Baader et al. 2003]. We remind the reader that description logics (DLs, for short) are knowledge representation and reasoning formalisms in which complex concepts are defined in terms of atomic concepts using certain constructors. DLs are then used to represent, and reason about, various relations between such complex concepts (typically, the subsumption relation). The basic Boolean description logic $\mathcal{A L C}$ has as its constructors the Boolean connectives and the universal restriction $\forall r$, which, for a concept $C$ and a binary relation symbol $r$, gives the concept $\forall r . C$ containing precisely those objects $x$ from the underlying domain for which $y \in C$ whenever $x r y$. The language $\mathcal{A L C}$ is a notational variant of the basic modal logic $\mathrm{K}$ with infinitely many modal operators: propositional variables correspond to atomic concepts, while $\forall r . C$ is interpreted in a relational structure in the same way as $\square_{r}$ (the modal box interpreted by the accessibility relation $r$ ). We refer the reader to [Baader et al. 2003] for precise definitions and a discussion of syntax and semantics of $\mathcal{A L C}$ and other description logics.

It has been argued in [Baader and Narendran 2001] that for many applications of DLs it would be useful to have an algorithm capable of deciding, given two complex concepts $C_{1}$ and $C_{2}$, whether there exists a substitution $s$ (of possibly complex concepts in place of atomic ones) such that $s\left(C_{1}\right)$ is equivalent to $s\left(C_{2}\right)$ in the given DL. ${ }^{3}$ We call this problem the concept unification problem. A typical application of such an algorithm is as follows. In many cases, knowledge bases (ontologies) based on DLs are developed by different knowledge engineers over a long period. It can therefore happen that some concepts which, intuitively, should be equivalent, are introduced several times with slightly different definitions. To detect such redundancies, one can check whether certain concepts can be unified. Unifiability does not necessarily mean that these concepts have indeed been defined to denote the same class of objects-but this fact can serve as an indicator of a possible redundancy, so that the knowledge engineer could then 'double check' the meaning of those concepts and change the knowledge base accordingly.

The concept unification problem for $\mathcal{A L C}$ is easily seen to be equivalent to the unification problem for the modal logic $\mathrm{K}$ with infinitely many modal operators: formulated for the modal language, the problem is to decide whether, given two modal formulas $\varphi_{1}$ and $\varphi_{2}$, there exists a substitution $s$ such that, for every Kripke model $\mathfrak{M}$ and every point $x$ in it,

$$
(\mathfrak{M}, x) \models s\left(\varphi_{1}\right) \quad \text { iff } \quad(\mathfrak{M}, x) \models s\left(\varphi_{2}\right) .
$$

This is obviously equivalent to the validity of $s\left(\varphi_{1} \leftrightarrow \varphi_{2}\right)$. Baader and Kuesters [2001] and Baader and Narendran [2001] develop decision procedures for the concept unification problem for a number of sub-Boolean DLs, that is, DLs which do not have all the Boolean connectives as constructors and are, therefore, either properly less expressive than $\mathcal{A L C}$ or incomparable with $\mathcal{A L C}$. The investigation of the

\footnotetext{
${ }^{3}$ This is the simplest version of the decision problem they consider. More generally, Baader and Narendran [2001] consider the problem whether there exists such a substitution which leaves certain atomic concepts intact. We will not consider this more complex decision problem in this paper.
} 
concept unification problem for Boolean DLs, that is, $\mathcal{A L C}$ and its extensions, is left as an open research problem.

It should be clear that we have to leave open the decidability status for the concept unification problem for $\mathcal{A L C}$ as well. However, we obtain the undecidability of this problem for extensions of $\mathcal{A L C}$ with nominals. In contemporary description logic research and applications, nominals play a major role, see e.g., [Horrocks and Sattler 2005] and references therein. The smallest description logic containing $\mathcal{A L C}$ and nominals is known as $\mathcal{A L C O}$, and by extending the mapping between modal and description languages indicated above, one can see that $\mathcal{A L C O}$ is a straightforward notational variant of the modal logic with infinitely many modal operators and nominals. Hence, as a consequence of Theorem 4.2 we obtain:

TheOREM 5.1. The concept unification problem for $\mathcal{A L C O}$ is undecidable.

Moreover, the undecidability proof goes through as well for extensions of $\mathcal{A L C O}$ such as, for example, $\mathcal{A L C} \mathcal{Q O}$ and $\mathcal{S H I} \mathcal{I O}$, the description logic underlying OWLDL [Horrocks et al. 2003].

Another family of description logics for which the concept unification problem turns out to be undecidable are those extensions of $\mathcal{A L C}$ where the universal role is definable. The minimal description logic of this sort, widely used in DL applications, is known nowadays as $\mathcal{S H \mathcal { I }}$. Originally, Horrocks and Sattler [1999] introduced this logic under the name $\mathcal{A} \mathcal{L C H} \mathcal{I}_{R^{+}}$. In $\mathcal{S H I}$, the signature of $\mathcal{A L C}$ is extended by

-infinitely many relation symbols, which are interpreted by transitive relations,

- and for each relation symbol $r$, there is a relation symbol $r^{-}$, which is interpreted by the inverse of the interpretation of $r$.

The concept unification problem for $\mathcal{S H I}$ remains open. However, when considering $\mathcal{S H I}$ it is not the concept unification problem one is mainly interested in, but its generalisation to the concept unification relative to role axioms ${ }^{4}$ : in $\mathcal{S H I}$ and its extensions one can state in a so-called RBox (role box) that the interpretation of a relation symbol $r$ is included in the interpretation of a relation symbol $s$, in symbols $r \sqsubseteq s$. Now, $\mathcal{S H I}$ concepts $C$ and $D$ are called unifiable relative to an RBox $R$ iff

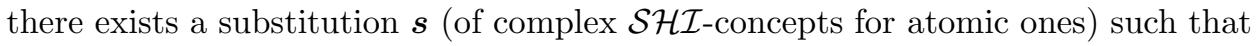
$s(C)$ is equivalent to $s(D)$ in every model satisfying the RBox $R$. It easily seen that this problem is undecidable. Indeed, consider the RBox $R$ consisting of $s \sqsubseteq s^{-}$, $s^{-} \sqsubseteq s$, and $r \sqsubseteq s$, where $s$ is a transitive role. Then, in every model for $R, s$ is transitive, symmetric and contains $r$. By replacing the operator $\square$ with $\forall r$ and the operator $\forall$ with $\forall s$ in the proof of Theorem 2.3, one can easily show that concept unification relative to the RBox $R$ is undecidable. Thus we obtain the following:

THEOREM 5.2. The concept unification problem relative to role axioms for $\mathcal{S H \mathcal { I }}$ is undecidable.

This undecidability proof also goes through for extensions of $\mathcal{S H \mathcal { I }}$ such as, for example, $\mathcal{S H \mathcal { I N }}$ and $\mathcal{S H I} \mathcal{Q}$.

${ }^{4}$ In description logic, the most useful generalisation of the concept unification problem is unification relative to TBoxes and RBoxes. We will not discuss this generalisation here because the undecidability results presented in this paper trivially hold for it as well.

ACM Transactions on Computational Logic, Vol. V, No. N, June 2021. 


\section{CONCLUSION}

In this paper, we have shown that for two standard constructors of modal logic - the universal modality and nominals - the unification and admissibility problems are undecidable. It follows that both unification and admissibility are undecidable for all standard hybrid logics and many of the most frequently employed description logics.

Many intriguing problems remain open. The question whether the unification and admissibility problems for $\mathrm{K}$ (or, equivalently, $\mathcal{A L C}$ ) are decidable is one of the major open problems in modal and description logic.

\section{ACKNOWLEDGMENTS}

We were partially supported by the U.K. EPSRC grants GR/S61966, GR/S63182, GR/S63175, GR/S61973.

\section{REFERENCES}

Areces, C., Blackburn, P., And Marx, M. 2000. The computational complexity of hybrid temporal logics. Logic Journal of IGPL 8, 653-679.

Areces, C. and ten Cate, B. 2006. Hybrid logics. See Blackburn et al. [2006], 821-867.

Baader, F., Calvanese, D., McGuinness, D., Nardi, D., and Patel-Schneider, P., Eds. 2003. The Description Logic Handbook: Theory, Implementation, and Applications. Cambridge University Press.

BAADER, F. AND Küsters, R. 2001. Unification in a description logic with transitive closure of roles. In Proceedings of the 8th International Conference on Logic for Programming, Artificial Intelligence, and Reasoning (LPAR 2001), R. Nieuwenhuis and A. Voronkov, Eds. Lecture Notes in Computer Science, vol. 2250. Springer-Verlag, Havana, Cuba, 217-232.

BAADER, F. AND NAREndRAn, P. 2001. Unification of concept terms in description logics. Journal of Symbolic Computation 31, 277-305.

BaAder, F. And Siekmann, J. 1994. Unification theory. In Handbook of Logic in Artificial Intelligence and Logic Programming, D. Gabbay, C. Hogger, and J. Robinson, Eds. Oxford University Press.

BaAder, F. And SNyder, W. 2001. Unification theory. In Handbook of Automated Reasoning, J. Robinson and A. Voronkov, Eds. Vol. I. Elsevier Science Publishers, 447-533.

Blackburn, P., van Benthem, J., And Wolter, F., Eds. 2006. Handbook of Modal Logic. Elsevier.

Chagrov, A. 1990. Undecidable properties of extensions of provability logic. I. Algebra and Logic 29, 231-243.

Chagrov, A. 1992. A decidable modal logic with the undecidable admissibility problem for inference rules. Algebra and Logic 31, 53-61.

Chagrov, A. And Zakharyaschev, M. 1997. Modal Logic. Oxford Logic Guides, vol. 35. Clarendon Press, Oxford.

Ebbinghaus, H.-D., Flum, J., And Thomas, W. 1994. Mathematical Logic. Springer.

Ghilardi, S. 2000. Best solving modal equations. Annals of Pure and Applied Logic 102, 183-198.

GHILARDI, S. 2004. Unification, finite duality and projectivity in locally finite varieties of Heyting algebras. Annals of Pure and Applied Logic 127, 99-115.

Ghilardi, S. And SACChetti, L. 2004. Filtering unification and most general unifiers in modal logic. Journal of Symbolic Logic 69, 879-906.

Goranko, V. AND PAssy, S. 1992. Using the universal modality: Gains and questions. Journal of Logic and Computation 2, 5-30.

Harel, D., Kozen, D., and Tiuryn, J. 2000. Dynamic Logic. Mit Press.

Horrocks, I., Patel-Schneider, P. F., and van Harmelen, F. 2003. From $\mathcal{S H \mathcal { I }}$ and RDF to OWL: The making of a web ontology language. Journal of Web Semantics 1, 7-26. 
Horrocks, I. And Sattler, U. 1999. A description logic with transitive and inverse roles and role hierarchies. Journal of Logic and Computation 9, 385-410.

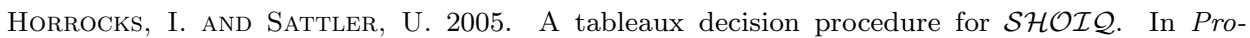
ceedings of Nineteenth International Joint Conference on Artificial Intelligence (IJCAI 2005), L. Kaelbling and A. Saffiotti, Eds. Professional Book Center, 448-453.

IEмноғF, R. 2001. On the admissible rules of intuitionistic propositional logic. Journal of Symbolic Logic 66, 281-294.

IEmhoff, R. 2003. Towards a proof system for admissibility. In Proceedings of the 17th International Workshop 'Computer Science Logic', M. Baaz and J. Makowsky, Eds. Lecture Notes in Computer Science, vol. 2803. Springer, 255-270.

Minsky, M. 1961. Recursive unsolvability of Post's problem of "tag" and other topics in the theory of Turing machines. Annals of Mathematics 74, 437-455.

Rybakov, V. 1997. Admissibility of Logical Inference Rules. Studies in Logic and the Foundations of Mathematics, vol. 136. Elsevier.

Venema, Y. 2006. Algebras and coalgebras. See Blackburn et al. [2006], 331-425.

Zakharyaschev, M., Wolter, F., And Chagrov, A. 2001. Advanced modal logic. In Handbook of Philosophical Logic, 2nd edition, D. Gabbay and F. Guenthner, Eds. Vol. 3. Kluwer Academic Publishers, 83-266.

ACM Transactions on Computational Logic, Vol. V, No. N, June 2021. 